\title{
Inorganic versus organic semiconductors
}

\section{Editorial}

Generally, inorganic semiconductors are categorized into elemental and compound semiconductors. The lattice of elemental inorganic semiconductors consists of only a single type of atom and they are found in IV and VI groups of the periodic table. The most popular elemental inorganic semiconductors are $\mathrm{Si}$ and $\mathrm{Ge}$. The lattice of compound inorganic semiconductors commonly contains two or more species of atoms. They usually combine elements either from III and $\mathrm{V}$ or II and VI groups of the periodic table. The prominent examples of these materials are gallium arsenide, gallium nitride, indium gallium nitride, zinc selenide and so on. OSCs and inorganic semiconductors differ substantially from the point of view of energy states which have an essential influence on charge transport properties of a material. The increase in the operational temperature generally leads to the improvement of the electrical behavior of organic semiconductors (OSCs) as high temperature causes lattice vibrations and provides required thermal energy for the system to create favourable environments for the release of the trapped charge carriers. ${ }^{1-3}$ The charge transport mechanism of inorganic semiconductors is sensitive to temperature as well. However, it is extremely detrimental to the performance of these materials as the temperature-induced lattice vibrations of the highly-ordered and crystalline inorganic substances lead to increased lattice scattering and trapping of the charges as well as reduced carrier mobility. ${ }^{4}$

OSCs display disordered non-crystalline amorphous arrangements with a large number of localized states (traps) randomly distributed over the lattice..$^{5}$ In addition, the structural disorder present in the organic molecules results in the energetic disorder of their molecules which is the origin of the low charge carrier mobility and conductivity. ${ }^{5}$ Polymers are intrinsically highly amorphous macromolecules with flexible backbones. However, semi-crystallinity has also been observed in some regio-regular polymeric semiconductors such as poly(3-hexylthiophene) (P3HT) and poly(2,5-bis-(3-alkylthiophen2-yl)thieno[3,2-b]thiophene) (PBT). ${ }^{6}$ Compared to completely amorphous polymers, higher crystallinity in these semi-crystalline molecules induces higher charge carrier mobility as hoppingassisted charge transport between $\pi$-stacked crystalline domains and longer polymer chains formed by neighboring polymer crystallites, occurs longitudinally with a smaller number of obstacles. Unlike polymers, typical small molecular OSCs possess relatively ordered and crystalline structures resulting from their rigid molecular backbones. Therefore, most small molecules outperform widely studied polymers in electrical conductivity. However, just like in polymers, the parts of the small molecules are also bonded by weak van der Waals forces and are sensitive to external factors including oxygen molecules existing in the ambient environment. Examples of crystalline OSCs primarily include pentacene, anthracene, rubrene, 3,4,9,10-perylenetetracarboxylic dianhydride (PTCDA), 1,4,5,8-naphthalenetetracarboxylic dianhydride (NTCDA), phthalocyanines, fullerenes, and other aromatic compounds.

Studies have shown that doping efficiency of OSCs is strongly dependent on the structure of the used host materials. For instance, doping of solution-processed polymers is highly dependent on the batch-to-batch synthesis variations in their molecular weight

\author{
Volume 4 Issue 6 - 2020
}

Gunel Huseynova

RIPE \& 3D Printing, Hanbat National University, South Korea

Correspondence: Gunel Huseynova, RIPE \& 3D Printing, Hanbat National University, 34I58, South Korea, Tel +4672778207l, Email bblg739@gmail.com

Received: December 30, 2020 | Published: December 31 , 2020

distribution as well as on the variation of their film morphology and microstructural properties including the symmetry and planarity of the polymer backbones, $\pi-\pi$ stacking between the chains and their molecular packing, etc., which are strongly dependent on the versatility of the used processing technique and environmental conditions including the post-annealing temperature of the polymer films. On the contrary, small molecular OSCs demonstrate outstanding batchto-batch regularity and uniform film forming properties due to their solid-state deposition techniques limited to specific high vacuum and low-pressure requirements. Small molecules are also easily purified to remove any possible residual interfering molecules or impurities. Therefore, doping of small molecules tends to demonstrate a higher potential to justify expectations and produce more reliable results compared to polymeric semiconductors. The studies focused on the doping effects of the fluorinated tetracyanoquinodimethane (TCNQ) derivatives on both vacuum-deposited small molecules and solutionprocessed polymers are probably the best examples revealing how these two different types of OSCs react to intentional impurities. Although majority of the TCNQ derivatives easily form charge transfer complexes (CTCs) with a variety of OSCs due to their strong electron accepting nature, the extent of their doping efficiencies varies considerably from material to material and is dependent on the structural properties and processing techniques of the used hosts. For instance, recent investigations have revealed that addition of 2,5-difluoro-7,7,8,8-tetracyanoquinodimethane (F2-TCNQ) can lead to enormously increased electrical conductivities and metallic transport behaviors in a wide range of different highly-ordered organic crystalline semiconductors while the same dopant and even famous F4-TCNQ which belongs to the same dopant category and is a stronger electron acceptor compared to $\mathrm{F} 2-\mathrm{TCNQ}$, increases the conductivity of polymeric semiconductors only by a few orders of magnitude. In addition, the effect of the dopants also varies from polymer to polymer yielding an individual doping profile for every distinct polymer. This phenomenon related to the unpredictable and distinctive dopant strength of the same dopants in different organic systems, is attributed to the structural and morphological differences of the used host molecules. ${ }^{5,7,8}$

\section{Acknowledgments}

\section{None.}




\section{Conflicts of interest}

The authors declare that there is no conflict of interest.

\section{References}

1. Samuele Giannini, Antoine Carof, Jochen Blumberger. Crossover from Hopping to Band-Like Charge Transport in an Organic Semiconductor Model: Atomistic Nonadiabatic Molecular Dynamics Simulation. J Phys Chem Lett. 2018;9(11):3116-3123.

2. Li W, Kwok H. Conduction Mechanisms in Organic Semiconductors. In Bhushan B, editor. Encyclopedia of Nanotechnology. Springer, Dordrecht. 2012.

3. Baranovskii S, Rubel O. Charge Transport in Disordered Materials. In: Kasap S, Capper P, editors. Springer Handbook of Electronic and Photonic Materials. Springer. 2017.
4. Schlettwein D. Electronic Properties of Molecular Organic Semiconductor Thin Films. Supramolecular Photosensitive and Electroactive Materials. 2001;211-338.

5. Kowalsky W, Rompf C. Crystalline Organic Semiconductors: A New Class of Materials for Photonic Devices. In: Ehrfeld W, Wegner G, editors. Integrated Optics and Micro-Optics with Polymers. TEUBNER-TEXTE zur Physik. Vieweg+Teubner Verlag, Wiesbaden. 1993.

6. Yim KH, Whiting GL, Murphy GE, et al. Controlling Electrical Properties of Conjugated Polymers via a Solution-Based p-Type Doping. Adv Mater. 2008;20(17):3319-3324.

7. Méndez H, Heimel G, Winkler S, et al. Charge-Transfer Crystallites as Molecular Electrical Dopants. Nat Commun. 2015;6:8560.

8. Shimada T, Takahashi Y, Harada J, et al. Band-Like Carrier Transport at the Single-Crystal Contact Interfaces between 2,5-Difluoro-7,7,8,8tetracyanoquinodimethane and Electron Donors. J Phys Chem. Lett. 2018;9:420-424. 